\title{
MÜNTZ SYSTEMS AND ORTHOGONAL MÜNTZ-LEGENDRE POLYNOMIALS
}

\author{
PETER BORWEIN, TAMÁS ERDÉLYI, AND JOHN ZHANG
}

\begin{abstract}
The Müntz-Legendre polynomials arise by orthogonalizing the Müntz system $\left\{x^{\lambda_{0}}, x^{\lambda_{1}}, \ldots\right\}$ with respect to Lebesgue measure on $[0,1]$. In this paper, differential and integral recurrence formulae for the Müntz-Legendre polynomials are obtained. Interlacing and lexicographical properties of their zeros are studied, and the smallest and largest zeros are universally estimated via the zeros of Laguerre polynomials. The uniform convergence of the Christoffel functions is proved equivalent to the nondenseness of the Müntz space on $[0,1]$, which implies that in this case the orthogonal Müntz-Legendre polynomials tend to 0 uniformly on closed subintervals of $[0,1)$. Some inequalities for Müntz polynomials are also investigated, most notably, a sharp $L^{2}$ Markov inequality is proved.
\end{abstract}

\section{INTRODUCTION}

Let $0 \leq \lambda_{0}<\lambda_{1}<\cdots \rightarrow \infty$. The classical Müntz-Szász Theorem states that the Müntz polynomials of the form $\sum_{k=0}^{n} a_{k} x^{\lambda_{k}}$ with real coefficients are dense in $L^{2}[0,1]$ if and only if

$$
\sum_{k=1}^{\infty} \lambda_{k}^{-1}=+\infty .
$$

If the constant function 1 is also in the system, that is, $\lambda_{0}=0$, then the denseness of the Müntz polynomials in $C[0,1]$ in the uniform norm is also characterized by (1.1). It is our intention to examine various facets of the Müntz space

$$
M=\operatorname{span}\left\{x^{\lambda_{0}}, x^{\lambda_{1}}, x^{\lambda_{2}}, \ldots\right\}
$$

and for its subspaces

$$
M_{n}=\operatorname{span}\left\{x^{\lambda_{0}}, x^{\lambda_{1}}, \ldots, x^{\lambda_{n}}\right\},
$$

where the span is taken over all real numbers ( $\S \S 4$ and 5, where real properties are studied) or complex numbers ( $\S \S 2$ and 3 ). It has been observed [25, MR

Received by the editors January 10, 1992.

1991 Mathematics Subject Classification. Primary 41A17, 42C05; Secondary 30C15, 39A10.

Key words and phrases. Exponential polynomials, Müntz systems, Müntz-Szász Theorem, MüntzLegendre polynomials, recurrence formulae, orthogonal polynomials, Christoffel functions, Markovtype inequalities, Bernstein-type inequalities, Nikolskii-type inequalities, interlacing properties of zeros, lexicographic properties of zeros.

Research supported by NSERC and NSF Grant DMS- 9024901. 
\#88e:33008] and [13], but does not appear to be particularly well known, that the orthogonal polynomials associated with a Müntz system (with respect to Lebesgue measure) on $[0,1]$ can be explicitly written down. These orthogonal polynomials are called Müntz-Legendre polynomials. This is the key tool for the analysis we undertake. We prove for example the $L^{2}$ Markov inequality

$$
\frac{\left\|x p^{\prime}(x)\right\|_{2}}{\|p\|_{2}} \leq \frac{1}{\sqrt{2}} \sum_{k=0}^{n}\left(1+2 \lambda_{k}\right)
$$

for all Müntz polynomials $p$ from $M_{n}$. Compare this with the $L^{\infty}$ result in [17]

$$
\frac{\left\|x p^{\prime}(x)\right\|_{\infty}}{\|p\|_{\infty}} \leq 11 \sum_{k=0}^{n} \lambda_{k} .
$$

Both of these are sharp up to the constants. In order to prove this result and various of its relatives we first derive some explicit formulae and recursions for the sequence of Müntz-Legendre polynomials. Since this orthogonalization is not well known, and for the sake of completeness, we briefly reprove some of the basic formulae, some of which may be found in $[13,25]$. This is contained in $\S 2$. Section 3 offers some inequalities for Müntz polynomials, mainly, the above-mentioned $L^{2}$ Markov inequality. In $\S 4$, we study the interlacing and lexicographical properties of the zeros of Müntz-Legendre polynomials. Also in this section, universal estimates of the smallest and largest zeros of MüntzLegendre polynomials are obtained via the zeros of Laguerre polynomials. Finally in the last section, we study the properties of the Christoffel functions, whose pointwise or uniform convergence on closed subintervals of $[0,1)$ turns out to give a characterization of the nondenseness of the Müntz space on $[0,1]$.

Proofs of the Müntz-Szász Theorem can be found in [6, 8, and 10], and various new developments are in $[1-5,7,8,11,17,18,21-23,26]$. A very special class of Müntz systems, the incomplete polynomials of the form $x^{m} p(x)$ with ordinary polynomials $p$ has been studied intensively (cf. $[12,20]$ ).

\section{BASIC PROPERTIES OF MÜNTZ-LEGENDRE POLYNOMIALS}

Throughout this paper, we adopt the following definition for $x^{\lambda}$ :

$$
x^{\lambda}=e^{\lambda \log x}, \quad x \in(0, \infty), \lambda \in \mathbb{C},
$$

and the value at $x=0$ is defined to be the limit of $x^{\lambda}$ as $x \rightarrow 0$ from $(0, \infty)$ whenever the limit exists. Given a complex sequence $\Lambda=\left\{\lambda_{0}, \lambda_{1}, \lambda_{2}, \ldots\right\}$, a linear combination of the Müntz system $\left\{x^{\lambda_{0}}, x^{\lambda_{1}}, \ldots, x^{\lambda_{n}}\right\}$ is called a Müntz polynomial, or a $\Lambda$-polynomial. Denote the set of all such polynomials by $M_{n}(\Lambda)$, that is,

$$
M_{n}(\Lambda)=\operatorname{span}\left\{x^{\lambda_{0}}, x^{\lambda_{1}}, \ldots, x^{\lambda_{n}}\right\},
$$

where the linear span is over the real numbers $(\S 4$ and $\S 5)$ or over the complex numbers (this section and $\S 3$ ), according to context. The union of all $M_{n}(\Lambda)$ is denoted by $M(\Lambda)$, that is,

$$
M(\Lambda)=\bigcup_{n=0}^{\infty} M_{n}(\Lambda)
$$


For the $L^{2}$ theory of a Müntz system, we consider

$$
\Lambda=\left\{\lambda_{0}, \lambda_{1}, \lambda_{2}, \ldots\right\}, \quad \mathfrak{R}\left(\lambda_{k}\right)>-1 / 2, \text { and } \lambda_{k} \neq \lambda_{j}(k \neq j),
$$

where $\mathfrak{R}(\lambda)$ is the real part of $\lambda$. This ensures that every $\Lambda$-polynomial is in $L^{2}[0,1]$. We can then define the orthogonal $\Lambda$-polynomials with respect to the Lebesgue measure, the Müntz-Legendre polynomials. Although we almost always assume (2.4), the following definition does not require the distinctness of the exponents $\lambda_{k}$.

Definition 2.1. Let $\Lambda=\left\{\lambda_{0}, \lambda_{1}, \lambda_{2}, \ldots\right\}$ be a complex sequence. We define the $n$th Müntz-Legendre polynomial on $(0,1]$ to be (cf. [25])

$$
L_{n}\left(\lambda_{0}, \ldots, \lambda_{n} ; x\right)=\frac{1}{2 \pi i} \int_{\Gamma} \prod_{k=0}^{n-1} \frac{t+\bar{\lambda}_{k}+1}{t-\lambda_{k}} \frac{x^{t} d t}{t-\lambda_{n}}, \quad n=0,1,2, \ldots,
$$

where the simple contour $\Gamma$ surrounds all the zeros of the denominator in the integrand, and $\bar{\lambda}$ denotes the conjugate of $\lambda$.

The orthogonality of the above functions with respect to the Lebesgue measure will be proved in Corollary 2.3. Here we first record an immediate consequence of the definition and the Residue Theorem.

Corollary 2.2. Let $\Lambda=\left\{\lambda_{0}, \lambda_{1}, \lambda_{2}, \ldots\right\}$ satisfy (2.4). Then for every $n=$ $0,1,2, \ldots$,

$$
L_{n}\left(\lambda_{0}, \ldots, \lambda_{n} ; x\right)=\sum_{k=0}^{n} c_{k, n} x^{\lambda_{k}}, \quad c_{k, n}=\frac{\prod_{j=0}^{n-1}\left(\lambda_{k}+\bar{\lambda}_{j}+1\right)}{\prod_{j=0, j \neq k}^{n}\left(\lambda_{k}-\lambda_{j}\right)}
$$

with $L_{n}\left(\lambda_{0}, \ldots, \lambda_{n} ; x\right)$ defined by $(2.5)$.

So, $L_{n}\left(\lambda_{0}, \ldots, \lambda_{n}\right)$ is indeed a $\Lambda$-polynomial provided that $\lambda_{0}, \lambda_{1}, \ldots, \lambda_{n}$ are distinct. Its value at $x=0$ is defined if for all $k$ either $\mathfrak{R}\left(\lambda_{k}\right)>0$ or $\lambda_{k}=0$. For example, if $\lambda_{0}=0$ and $\Re\left(\lambda_{k}\right)>0(1 \leq k \leq n)$, then $L_{n}\left(\lambda_{0}, \ldots, \lambda_{n} ; 0\right)=c_{0, n}$, and it is 0 if $\Re\left(\lambda_{0}\right)>0$ also holds.

Remark. From either Definition 2.1 or Corollary 2.2, it is obvious that in $L_{n}\left(\lambda_{0}, \ldots, \lambda_{n}\right)$, the order of $\lambda_{0}, \ldots, \lambda_{n-1}$ does not make any difference, as long as $\lambda_{n}$ is kept last. For example, $L_{2}\left(\lambda_{0}, \lambda_{1}, \lambda_{2}\right)=L_{2}\left(\lambda_{1}, \lambda_{0}, \lambda_{2}\right)$, but both are usually different from $L_{2}\left(\lambda_{0}, \lambda_{2}, \lambda_{1}\right)$. For a fixed (ordered) sequence $\Lambda$, we will use $L_{n}(\Lambda)$, or simply $L_{n}$ to denote the $n$th Müntz-Legendre polynomial $L_{n}\left(\lambda_{0}, \ldots, \lambda_{n}\right)$, whenever there is no ambiguity.

In (2.6), repeated indices (for example, $\lambda_{0}=\lambda_{1}$ ) cause a problem. But in the original definition, $\lambda_{k}=\lambda_{j}$ is allowed. We can view this also as a limiting case $\left(\lambda_{k} \rightarrow \lambda_{j}\right)$. We state a very special case when all indices are the same, which turns out to be closely related to the Laguerre polynomials. Notice also that the result is actually no longer a $\Lambda$-polynomial, with $\log x$ coming into the picture.

Corollary 2.3. Let $L_{n}\left(\lambda_{0}, \ldots, \lambda_{n} ; x\right)$ be defined by (2.5). If $\lambda_{0}=\cdots=\lambda_{n}=\lambda$, then

$$
L_{n}\left(\lambda_{0}, \ldots, \lambda_{n} ; x\right)=x^{\lambda} \mathscr{L}_{n}(-(1+\lambda+\bar{\lambda}) \log x)
$$


where $\mathscr{L}_{n}$ is the nth Laguerre polynomial orthogonal with respect to the weight $e^{-x}$ on $[0, \infty)$ and with $\mathscr{L}_{n}(0)=1$.

Proof. Since $\lambda_{k}=\lambda$ for $k=0,1, \ldots, n,(2.5)$ yields

$$
L_{n}\left(\lambda_{0}, \lambda_{1}, \ldots, \lambda_{n} ; x\right)=\frac{1}{2 \pi i} \int_{\Gamma} \frac{x^{t}(t+\bar{\lambda}+1)^{n}}{(t-\lambda)^{n+1}} d t,
$$

where the contour $\Gamma$ can be taken to be any circle centered at $\lambda$. By the Residue Theorem,

$$
\begin{aligned}
L_{n}\left(\lambda_{0}, \ldots, \lambda_{n} ; x\right) & =\frac{d^{n}}{n ! d t^{n}}\left[x^{t}(t+\bar{\lambda}+1)^{n}\right]_{t=\lambda} \\
& =\frac{1}{n !} \sum_{k=0}^{n}\left(\begin{array}{l}
n \\
k
\end{array}\right) x^{\lambda}(\log x)^{k} n(n-1) \cdots(k+1)(\lambda+\bar{\lambda}+1)^{k} \\
& =x^{\lambda} \sum_{k=0}^{n} \frac{1}{k !}\left(\begin{array}{l}
n \\
k
\end{array}\right)(1+\lambda+\bar{\lambda})^{k} \log ^{k} x .
\end{aligned}
$$

These are just the Laguerre polynomials $\left\{\mathscr{L}_{n}\right\}$ in $(-\log x)$ which are orthogonal with respect to the weight function $e^{-t}$ on $[0, \infty)$ with the normalization $\mathscr{L}_{n}(0)=1$ (cf. [24, p. 100]), and we obtain (2.7).

The name Müntz-Legendre polynomial is justified by the following theorem, where the orthogonality of $\left\{L_{n}\right\}$ with respect to the Lebesgue measure is proved.

Theorem 2.4. Let $\Lambda=\left\{\lambda_{0}, \lambda_{1}, \lambda_{2}, \ldots\right\}$ satisfy $\mathfrak{R}\left(\lambda_{k}\right)>-1 / 2$ for $k=0,1$, $2, \ldots$. Assume that $L_{n}$ is defined by (2.5). Then

$$
\int_{0}^{1} L_{n}(x) \overline{L_{m}(x)}=\delta_{n, m} /\left(1+\lambda_{n}+\bar{\lambda}_{n}\right)
$$

holds for every $m, n=0,1,2, \ldots$.

Remark. In the orthogonality (2.8), repeated indices are allowed.

Proof. We provide a proof here for the sake of completeness. It suffices to consider $0 \leq m \leq n$. Also, we just need to prove (2.8) for distinct indices, since from the definition in $(2.5), L_{n}\left(\lambda_{0}, \ldots, \lambda_{n} ; x\right)$ is uniformly continuous in $\lambda_{0}, \ldots, \lambda_{n}$ for $x$ in closed subintervals of $(0,1]$, and the nondistinct case is a limiting argument. Since $\mathfrak{R}\left(\lambda_{k}\right)>-1 / 2$, we can pick a contour $\Gamma$ in the integral (2.5) such that $\Gamma$ lies completely to the right of the vertical line $\mathfrak{R}(t)=-1 / 2$, and $\Gamma$ surrounds all zeros of the denominator. When $t \in \Gamma$, we have $\mathfrak{R}\left(t+\bar{\lambda}_{m}\right)>-1$, and $\int_{0}^{1} x^{t+\bar{\lambda}_{m}} d x=1 /\left(1+t+\bar{\lambda}_{m}\right)$, for every $m \geq 0$. Hence,

$$
\int_{0}^{1} L_{n}(x) \overline{x^{\lambda_{m}}} d x=\frac{1}{2 \pi i} \int_{\Gamma}^{n-1} \frac{t+\bar{\lambda}_{k}+1}{t-\lambda_{k}} \frac{d t}{\left(t-\lambda_{n}\right)\left(t+\bar{\lambda}_{m}+1\right)} .
$$

Notice that for $m<n$, the new term $t+\bar{\lambda}_{m}+1$ in the denominator can be cancelled, and for $m=n$ the new pole $-\left(\bar{\lambda}_{n}+1\right)$ is outside $\Gamma$, because $\mathfrak{R}\left(-\bar{\lambda}_{n}-1\right)<-1 / 2$. Changing the contour from $\Gamma$ to $|t|=R$ with $R>$ 
$\max \left\{\left|\lambda_{0}\right|+1, \ldots,\left|\lambda_{n}\right|+1\right\}$, we have for $0 \leq m \leq n$ that

$$
\begin{aligned}
\int_{0}^{1} L_{n}(x) \overline{x^{\lambda_{m}}} d x= & \frac{1}{2 \pi i} \int_{|t|=R} \prod_{k=0}^{n-1} \frac{t+\bar{\lambda}_{k}+1}{t-\lambda_{k}} \frac{d t}{\left(t-\lambda_{n}\right)\left(t+\bar{\lambda}_{n}+1\right)} \\
& -\frac{\delta_{m, n}}{-\bar{\lambda}_{n}-1-\lambda_{n}} \prod_{k=0}^{n-1} \frac{-\bar{\lambda}_{n}+\lambda_{k}}{-\bar{\lambda}_{n}-1-\lambda_{k}} .
\end{aligned}
$$

Letting $R \rightarrow \infty$, we see that the integral on the right-hand side is actually 0 , which gives

$$
\int_{0}^{1} L_{n}(x) \overline{x^{\lambda_{m}}} d x=\frac{\delta_{m, n}}{\bar{\lambda}_{n}+\lambda_{n}+1} \prod_{k=0}^{n-1} \frac{\bar{\lambda}_{n}-\lambda_{k}}{\bar{\lambda}_{n}+\lambda_{k}+1} .
$$

Now with (2.6), we have for $0 \leq m \leq n$ that

$$
\begin{gathered}
\int_{0}^{1} L_{n}(x) \overline{L_{m}(x)} d x=\int_{0}^{1} L_{n}(x) \sum_{k=0}^{m} \overline{c_{k, m} x^{\lambda_{k}}} d x \\
=\overline{c_{m, m}} \int_{0}^{1} L_{n}(x) x^{\bar{\lambda}_{m}} d x=\delta_{m, n} /\left(\lambda_{n}+\bar{\lambda}_{n}+1\right)
\end{gathered}
$$

where the last step comes from the formula for $c_{k, n}$ in (2.6).

An alternative and probably easier proof of orthogonality follows from (2.10) below, integration by parts and induction. Later we will see that $L_{n}(1)=1$. This can be viewed as the normalization for Müntz-Legendre polynomial $L_{n}$. Clearly, if we let

$$
L_{n}^{*}:=\left(1+\lambda_{n}+\bar{\lambda}_{n}\right)^{1 / 2} L_{n},
$$

then we get an orthonormal system, that is,

$$
\int_{0}^{1} L_{n}^{*}(x) \overline{L_{m}^{*}(x)} d x=\delta_{m, n}, \quad m, n=0,1,2, \ldots
$$

These $L_{n}^{*}, n=0,1,2, \ldots$, will be called orthonormal Müntz-Legendre polynomials.

There is also a kind of Rodrigues formula for the Müntz-Legendre polynomials [13]. Let

$$
p_{n}(x)=\sum_{k=0}^{n} x^{\lambda_{k}} / \prod_{j=0, j \neq k}^{n}\left(\lambda_{k}-\lambda_{j}\right)
$$

then

$$
L_{n}(x)=D_{\lambda_{0}} \cdots D_{\lambda_{n-1}} p_{n}(x),
$$

where the differential operators $D_{\lambda}$ are defined by $D_{\lambda} f(x)=x^{-\bar{\lambda}} \frac{d}{d x}\left(x^{1+\bar{\lambda}} f(x)\right)$. Notice also that $p_{n}$ and its first $n-1$ derivatives vanish at $x=1$ (cf. [13]). This formula follows easily from Corollary 2.2 .

Now we state the differential recurrence formulae for $\left\{L_{n}\right\}$.

Theorem 2.5. Assume that $\Lambda$ is a complex sequence satisfying $\mathfrak{R}\left(\lambda_{k}\right)>-1 / 2$ for all $k$. Then

$$
x L_{n}^{\prime}(x)-x L_{n-1}^{\prime}(x)=\lambda_{n} L_{n}(x)+\left(1+\bar{\lambda}_{n-1}\right) L_{n-1}(x),
$$


where $L_{n}, n=0,1,2, \ldots$, are the associated Müntz-Legendre polynomials defined by (2.5).

Proof. From (2.5), we get

$$
\frac{d}{d x}\left(x^{-\lambda_{n}} L_{n}(x)\right)=\frac{1}{2 \pi i} \int_{\Gamma} \frac{\prod_{k=0}^{n-2}\left(t+\bar{\lambda}_{k}+1\right)}{\prod_{k=0}^{n-1}\left(t-\lambda_{n}\right)}\left(t+\bar{\lambda}_{n-1}+1\right) x^{t-\lambda_{n}-1} d t .
$$

Multiplying both sides by $x^{\lambda_{n}+\bar{\lambda}_{n-1}+1}$, we obtain

$$
x^{\lambda_{n}+\bar{\lambda}_{n-1}+1}\left(x^{-\lambda_{n}} L_{n}(x)\right)^{\prime}=\frac{1}{2 \pi i} \int_{\Gamma} \frac{\prod_{k=0}^{n-2}\left(t+\bar{\lambda}_{k}+1\right)}{\prod_{k=0}^{n-1}\left(t-\lambda_{k}\right)}\left(t+\bar{\lambda}_{n-1}+1\right) x^{t+\bar{\lambda}_{n-1}} d t,
$$

and again by the definition of $L_{n-1}$ (cf. (2.5)),

$$
x^{\lambda_{n}+\bar{\lambda}_{n-1}+1}\left(x^{-\lambda_{n}} L_{n}(x)\right)^{\prime}=\left(x^{\bar{\lambda}_{n-1}+1} L_{n-1}(x)\right)^{\prime} .
$$

Simplifying by the product rule and dividing both sides by $x^{\bar{\lambda}_{n-1}}$, we get (2.10).

Corollary 2.6. Let a complex sequence $\Lambda$ satisfy (2.4), and let the associated Müntz-Legendre polynomials $L_{n}$ and the orthonormal Müntz-Legendre polynomials $L_{n}^{*}$ be defined by (2.5) and (2.9), respectively. Then

$$
\begin{aligned}
& x L_{n}^{\prime}(x)=\lambda_{n} L_{n}(x)+\sum_{k=0}^{n-1}\left(\lambda_{k}+\bar{\lambda}_{k}+1\right) L_{k}(x), \\
& x L_{n}^{*^{\prime}}(x)=\lambda_{n} L_{n}^{*}(x)+\sqrt{\lambda_{n}+\bar{\lambda}_{n}+1} \sum_{k=0}^{n-1} \sqrt{\lambda_{k}+\bar{\lambda}_{k}+1} L_{k}^{*}(x),
\end{aligned}
$$

and

$$
x L_{n}^{\prime \prime}(x)=\left(\lambda_{n}-1\right) L_{n}^{\prime}(x)+\sum_{k=0}^{n-1}\left(\lambda_{k}+\bar{\lambda}_{k}+1\right) L_{k}^{\prime}(x)
$$

for every $x \in(0, \infty)$.

Proof. The first equality (2.11) follows from Theorem 2.4 by writing $x L_{n}^{\prime}(x)-$ $x L_{0}^{\prime}(x)$ as a telescoping sum. From (2.11) and the relation

$$
L_{k}^{*}=\left(\lambda_{k}+\bar{\lambda}_{k}+1\right)^{1 / 2} L_{k}
$$

(cf. (2.9)), we get (2.12). Differentiating (2.11), we obtain (2.13).

The values and derivative values of the Müntz-Legendre polynomials at 1 can all be calculated. They are useful in locating the zeros of Müntz-Legendre polynomials (cf. §4).

Corollary 2.7. Let $L_{n}$ be the nth Müntz-Legendre polynomial defined by (2.5) (or by (2.6) from $\Lambda$ satisfying (2.4)), then

$$
L_{n}(1)=1, \quad L_{n}^{\prime}(1)=\lambda_{n}+\sum_{k=0}^{n-1}\left(\lambda_{k}+\bar{\lambda}_{k}+1\right), \quad n=0,1,2, \ldots,
$$


and

$$
L_{n}^{\prime \prime}(1)=\left(\lambda_{n}-1\right) L_{n}^{\prime}(1)+\sum_{k=0}^{n-1}\left(\lambda_{k}+\bar{\lambda}_{k}+1\right) L_{k}^{\prime}(1), \quad n=0,1,2, \ldots
$$

Proof. It suffices to show that $L_{n}(1)=1$, for the rest follows from Corollary 2.6. Notice that from (2.5),

$$
L_{n}(1)=\frac{1}{2 \pi i} \int_{\Gamma} \prod_{k=0}^{n-1} \frac{t+\bar{\lambda}_{k}+1}{t-\lambda_{n}} \frac{d t}{t-\lambda_{n}}
$$

Since $\Gamma$ surrounds all zeros of the denominator, and the degree of the denominator is one higher than that of the numerator, let $\Gamma$ be the circle $|t|=R$ and let $R \rightarrow \infty$. From this we get $L_{n}(1)=1$.

The recurrence formula can also be expressed in an integral form.

Corollary 2.8. Let a complex sequence $\Lambda$ satisfy (2.4), and let $L_{n}, n=0,1$, $2, \ldots$, be the Müntz-Legendre polynomials defined by (2.5). Then,

$$
L_{n}(x)=L_{n-1}(x)-\left(\lambda_{n}+\bar{\lambda}_{n-1}+1\right) x^{\lambda_{n}} \int_{x}^{1} x^{-\lambda_{n}-1} L_{n-1}(t) d t
$$

$$
x \in(0,1] \text {. }
$$

Proof. Rewriting the recurrence formula (2.10) as

$$
x L_{n}(x)-\lambda_{n} L_{n}(x)=x L_{n-1}^{\prime}(x)+\left(1+\bar{\lambda}_{n-1}\right) L_{n-1}(x),
$$

and multiplying both sides by $x^{-\lambda_{n}-1}$, we obtain

$$
\left(x^{-\lambda_{n}} L_{n}(x)\right)^{\prime}=x^{-\lambda_{n}} L_{n-1}^{\prime}(x)+\left(1+\bar{\lambda}_{n-1}\right) x^{-\lambda_{n}-1} L_{n-1}(x) .
$$

On taking the definite integral of the above on $[x, 1]$, and using the fact that $L_{k}(1)=1$ for all $k \geq 0$, we conclude

$$
\begin{aligned}
1-x^{-\lambda_{n}} L_{n}(x)= & 1-x^{-\lambda_{n}} L_{n-1}(x)-\int_{x}^{1}\left(t^{-\lambda_{n}}\right)^{\prime} L_{n-1}(t) d t \\
& +\left(\bar{\lambda}_{n-1}+1\right) \int_{x}^{1} t^{-\lambda_{n}-1} L_{n-1}(t) d t,
\end{aligned}
$$

which implies (2.16).

Another observation is that if $0 \leq \lambda_{n} \rightarrow \infty$ very fast, then $x=1$ is the unique maximal point of the Müntz-Legendre polynomial on $[0,1]$. A reasonable conjecture seems to be that the maximum of $\left|L_{n}\right|$ is always attained at one of the endpoints of $[0,1]$ when $\lambda_{n} \geq 0$, at least for regularly going sequences.

Corollary 2.9. If $\Lambda=\left\{\lambda_{0}, \lambda_{1}, \lambda_{2}, \ldots\right\}$ is a nonnegative sequence such that

$$
\lambda_{n} \geq \sum_{k=0}^{n-1}\left(1+2 \lambda_{k}\right), \quad n=1,2,3, \ldots
$$

then

$$
\left|L_{n}(x)\right|<L_{n}(1)=1, \quad x \in[0,1), n=2,3,4, \ldots \text {. }
$$

Remark. If $\lambda_{k}=\rho^{k}$, then (2.17) holds if and only if $\rho \geq 2+\sqrt{3}$. 
Proof. We assume $\lambda_{0}=0$. (The proof for $\lambda_{0}>0$ is essentially the same.) In this case, $L_{0}(x) \equiv 1$, and (2.18) fails for $n=0$. From (2.17), $\lambda_{1} \geq 1$, and $\lambda_{k} \geq 2+\lambda_{k-1}$ for $k \geq 2$. By (2.6),

$$
\left|L_{n}(0)\right|=\left|c_{0, n}\right|=\frac{\prod_{j=0}^{n-1}\left|1+\lambda_{j}\right|}{\prod_{j=1}^{n}\left|\lambda_{j}\right|}=\prod_{j=1}^{n} \frac{1+\lambda_{j-1}}{\lambda_{j}} .
$$

Hence, $\left|L_{1}(0)\right| \leq 1$, and $L_{n}(0)<1$ for every $n \geq 2$. Now we use induction to show that $\left|L_{n}(x)\right|<1$ on $(0,1)$ for every $n \geq 1$. Indeed, for $n=1$, because $\left|L_{1}(0)\right| \leq 1=L_{1}(1)$, and $L_{1}(x)=c_{0,1}+c_{1,1} x^{\lambda_{1}}$ is monotone on $[0,1]$, we have $\left|L_{1}(x)\right|<1$ on $(0,1)$. Assume that $n \geq 2$, and $\left|L_{k}(x)\right|<1$ for $1 \leq k \leq n-1$. Let $x$ be a local maximal point of $\left|L_{n}\right|$ in $(0,1)$, then $L_{n}^{\prime}(x)=0$. Hence Corollary 2.6 yields

Therefore

$$
\lambda_{n} L_{n}(x)+\sum_{k=0}^{n-1}\left(1+2 \lambda_{k}\right) L_{k}(x)=0 .
$$

$$
\left|L_{n}(x)\right|=\frac{1}{\lambda_{n}}\left|\sum_{k=0}^{n-1}\left(1+2 \lambda_{k}\right) L_{k}(x)\right|<\sum_{k=0}^{n-1}\left(1+2 \lambda_{k}\right) / \lambda_{n} \leq 1 .
$$

We finish this section by introducing the reproducing kernels. They are similar to the Dirichlet kernels in the trigonometric theory, or to the reproducing kernels for ordinary polynomials (cf. [24, p. 40]).

Corollary 2.10. Let $\Lambda=\left\{\lambda_{0}, \lambda_{1}, \lambda_{2}, \ldots\right\}$ be as in (2.4), and let $L_{n}$ and $L_{n}^{*}$ be defined by (2.5) and (2.9). Then for every $\Lambda$-polynomial $p(x)=\sum_{k=0}^{n} a_{k} x^{\lambda_{k}}$ in $M_{n}(\Lambda)$, we have

$$
p(x)=\int_{0}^{1} K_{n}(x, t) p(t) d t,
$$

where

$$
K_{n}(x, t)=\sum_{k=0}^{n} L_{k}^{*}(x) \overline{L_{k}^{*}(t)}
$$

is the nth reproducing kernel.

Proof. This is a well-known consequence of orthogonality. Since $L_{n}^{*}, n=$ $0,1,2, \ldots$, form an orthogonal system

$$
\int_{0}^{1} K_{n}(x, t) L_{k}^{*}(t) d t=L_{k}^{*}(x), \quad 0 \leq k \leq n .
$$

Note that $\left\{L_{0}^{*}, \ldots, L_{n}^{*}\right\}$ is a basis of $M_{n}(\Lambda)$, and the above is equivalent to (2.19).

Later in $\S 3$ and $\S 5$, we will see the importance of $K_{n}$ in solving an extremal problem for $\Lambda$-polynomials, and in the characterization of denseness of Müntz spaces.

\section{INEQUALITIES FOR MÜNTZ SYSTEMS}

Let $\Lambda=\left\{\lambda_{0}, \lambda_{1}, \lambda_{2}, \ldots\right\}$ satisfy (2.4) and let the Müntz spaces $M(\Lambda)$ and $M_{n}(\Lambda)$ be defined by (2.2) and (2.3). With the help of Müntz-Legendre polynomials, we establish some inequalities for $\Lambda$-polynomials. 
We now record an estimate of a $\Lambda$-polynomial $p$ and its derivative at a point $y \in(0,1]$ in terms of its $L^{2}$ norm $\left(\|p\|_{2}:=\left(\int_{0}^{1}|p(t)|^{2} d t\right)^{1 / 2}\right)$. First we state a more general theorem in terms of linear functionals.

Theorem 3.1. Suppose that $\Lambda$ satisfies (2.4) and that $L_{n}^{*}, n=0,1,2, \ldots$, are the orthonormal Müntz-Legendre polynomials. Then

$$
|\phi(p)| \leq\left[\sum_{k=0}^{n}\left|\phi\left(L_{k}^{*}\right)\right|^{2}\right]^{1 / 2}\|p\|_{2}
$$

for every linear functional $\phi$ defined on the Müntz space $M_{n}(\Lambda)$, and for every $p \in M_{n}(\Lambda)$. If $\phi \not \equiv 0$, then the equality holds if and only if $p(x)=$ const $\sum_{k=0}^{n} \overline{\phi\left(L_{k}^{*}\right)} L_{k}^{*}(x)$.

Proof. This is also a well-known consequence of the orthogonality of $L_{n}^{*}, n=$ $0,1,2, \ldots$ (cf. [24, p. 39], where $\phi(p)=p(x)$ is considered). To show (3.1) we write

$$
p(x)=\sum_{k=0}^{n} c_{k} L_{k}^{*}(x)
$$

with $\sum_{k=0}^{n}\left|c_{k}\right|^{2}=\|p\|_{2}^{2}$. Hence, by the linearity of $\phi$, we have

$$
\phi(p)=\sum_{k=0}^{n} c_{k} \phi\left(L_{k}^{*}\right)
$$

The theorem now follows from the Cauchy-Schwarz inequality.

If the linear functional is $\phi(p)=p^{(\nu)}(y)$ for some fixed $y \in(0,1]$ and fixed integer $\nu$, then the above becomes

Corollary 3.2. Suppose that $\Lambda$ satisfies (2.4) and that $L_{n}^{*}, n=0,1,2, \ldots$, are the orthonormal Müntz-Legendre polynomials. Then

$$
\left|p^{(\nu)}(y)\right| \leq\left[\sum_{k=0}^{n}\left|L_{k}^{*(\nu)}(y)\right|^{2}\right]^{1 / 2}\|p\|_{2}
$$

for every $\Lambda$-polynomial $p \in M_{n}(\Lambda), \nu=0,1,2, \ldots$, and $y \in(0,1]$. Equality holds if and only if $p(x)=c \sum_{k=0}^{n} \overline{L_{k}^{*(\nu)}(y)} L_{k}^{*}(x)$ for some constant $c$.

Remark. An equivalent expression of (3.2) is

$$
\left[\sum_{k=0}^{n}\left|L_{k}^{*(\nu)}(y)\right|^{2}\right]^{1 / 2}=\max \left\{\left|p^{(\nu)}(y)\right|: p \in M_{n}(\Lambda),\|p\|_{2}=1\right\} \text {. }
$$

On letting $n \rightarrow \infty$, this leads to

$$
\left[\sum_{k=0}^{\infty}\left|L_{k}^{*(\nu)}(y)\right|^{2}\right]^{1 / 2}=\sup \left\{\left|p^{(\nu)}(y)\right|: p \in M(\Lambda),\|p\|_{2}=1\right\}
$$

which may be finite or infinite. We will return to this in $\S 5$.

More explicit estimates than those in Corollary 3.2 can be obtained by combining Corollary 3.2 and Corollary 2.7. For simplicity, we only consider the cases $\nu=0$ and $\nu=1$, that is, we only state the estimates for $|p(y)|$ and $\left|p^{\prime}(y)\right|$ in terms of $\|p\|_{2}$ and the exponents $\lambda_{k}$. These are of the flavour of Nikolskii and Bernstein type inequalities. 
Corollary 3.3. Under the conditions of Corollary 3.2, we have

$$
\left|y^{1 / 2} p(y)\right| \leq\left[\sum_{k=0}^{n} 1+2 \mathfrak{R}\left(\lambda_{k}\right)\right]^{1 / 2}\|p\|_{2}
$$

and

$$
\left|y^{3 / 2} p^{\prime}(y)\right| \leq\left[\sum_{k=0}^{n}\left(1+2 \Re\left(\lambda_{k}\right)\right)\left|\lambda_{k}+\sum_{j=0}^{k-1}\left(1+2 \mathfrak{R}\left(\lambda_{j}\right)\right)\right|^{2}\right]^{1 / 2}\|p\|_{2}
$$

hold for every $p \in M_{n}(\Lambda)$ and $y \in(0,1]$.

Proof. When $y=1$, the above is a simple combination of Corollaries 2.7 and 3.3. For $0<y<1$, the scaling $x \rightarrow y x$ reduces the problem to the case $y=1$.

We now focus on one of our principal results, the $L^{2}$ Markov inequalities for Müntz polynomials, whose $L^{\infty}$ version is in [17].

Theorem 3.4. Assume that $\Lambda=\left\{\lambda_{0}, \lambda_{1}, \lambda_{2}, \ldots\right\}$ is given as in (2.4). Then,

$$
\sup _{p \in M_{n}(\Lambda)} \frac{\left\|x p^{\prime}(x)\right\|_{2}}{\|p\|_{2}} \leq\left[\sum_{j=0}^{n}\left|\lambda_{j}\right|^{2}+\sum_{j=0}^{n}\left(1+2 \mathfrak{R}\left(\lambda_{j}\right)\right) \sum_{k=j+1}^{n}\left(1+2 \mathfrak{R}\left(\lambda_{k}\right)\right)\right]^{1 / 2}
$$

If, in addition, $\Lambda$ consists of nonnegative real numbers, then

$$
\frac{1}{\sqrt{120}} \sum_{j=0}^{n} \lambda_{j} \leq \sup _{p \in M_{n}(\Lambda)} \frac{\left\|x p^{\prime}(x)\right\|_{2}}{\|p\|_{2}} \leq \frac{1}{\sqrt{2}} \sum_{j=0}^{n}\left(1+2 \lambda_{j}\right)
$$

where $n$ is an arbitrary nonnegative integer.

Remark. It is easy to see that the imaginary part of the exponents $\lambda_{j}$ does not affect the Markov factor as much as their real parts. For example, if $\lambda_{j}=i j$, where $i$ is the imaginary unit, then the Markov bound on the right-hand side of $(3.7)$ is $\left[\sum_{j=0}^{n}\left(j^{2}+n-j\right)\right]^{1 / 2}=O\left(n^{3 / 2}\right)$, while $\lambda_{j}=j$ results in $O\left(n^{2}\right)$.

Proof. Let $p \in M_{n}(\Lambda)$ be arbitrary, and $\|p\|_{2}=1$. Then $p(x)=\sum_{k=0}^{n} a_{k} L_{k}^{*}(x)$, and $\|p\|_{2}^{2}=\sum_{k=0}^{n}\left|a_{k}\right|^{2}=1$. Thus,

$$
x p^{\prime}(x)=\sum_{k=0}^{n} a_{k} x L_{k}^{* \prime}(x) .
$$

If we use the recurrence formula (2.12) for the terms $x L_{k}^{* \prime}(x)$ in the above and rearrange the sum, we get

$$
x p^{\prime}(x)=\sum_{j=0}^{n}\left[a_{j} \lambda_{j}+\sqrt{1+\lambda_{j}+\bar{\lambda}_{j}} \sum_{k=j+1}^{n} a_{k} \sqrt{1+\lambda_{k}+\bar{\lambda}_{k}}\right] L_{j}^{*}(x) .
$$


Hence,

$$
\begin{aligned}
\int_{0}^{1}\left|x p^{\prime}(x)\right|^{2} d x & =\sum_{j=0}^{n}\left|a_{j} \lambda_{j}+\sqrt{1+\lambda_{j}+\bar{\lambda}_{j}} \sum_{k=j+1}^{n} a_{k} \sqrt{1+\lambda_{k}+\bar{\lambda}_{k}}\right|^{2} \\
& \leq \sum_{j=0}^{n}\left|\sqrt{1+\lambda_{j}+\bar{\lambda}_{j}} \sum_{k=j}^{n} a_{k} \sqrt{1+\lambda_{k}+\bar{\lambda}_{k}}\right|^{2}
\end{aligned}
$$

Applying the Cauchy-Schwarz inequality for each term in the sum, and recalling that $\sum_{k=0}^{n}\left|a_{k}\right|^{2}=1$, we obtain

$$
\begin{aligned}
\int_{0}^{1}\left|x p^{\prime}(x)\right|^{2} d x & \leq \sum_{j=0}^{n}\left[\left|\lambda_{j}\right|^{2}+\left(1+\lambda_{j}+\bar{\lambda}_{j}\right) \sum_{k=j+1}^{n}\left(1+\lambda_{k}+\bar{\lambda}_{k}\right)\right] \\
& \leq \frac{1}{2}\left[\sum_{j=0}^{n}\left(1+2\left|\lambda_{j}\right|\right)\right]^{2} .
\end{aligned}
$$

The above proves (3.7) and the right half of (3.8). To prove the sharpness for the case that each $\lambda_{k}$ is nonnegative, we need to find a $\Lambda$-polynomial $p \neq 0$ in $M_{n}(\Lambda)$, such that

$$
\left\|x p^{\prime}\right\|_{2}^{2} \geq \frac{1}{120}\left(\sum_{k=0}^{n} \lambda_{k}\right)^{2}\|p\|_{2}^{2}
$$

Corollary 3.2 suggests that a possible candidate is $\sum_{k=0}^{n} \overline{L_{k}^{* \prime}(1)} L_{k}^{*}(x)$, and indeed, this works. However, a slight alternation makes the estimation easier. We consider

$$
p(x)=\sum_{k=0}^{n} \sqrt{\lambda_{k}}\left(\sum_{j=0}^{k} \lambda_{j}\right) L_{k}^{*}(x) .
$$

Since the system $\left\{L_{k}^{*}\right\}_{k=0}^{\infty}$ is orthonormal, we have

$$
\int_{0}^{1}|p(x)|^{2} d x=\sum_{k=0}^{n} \lambda_{k}\left(\sum_{j=0}^{k} \lambda_{j}\right)^{2} \leq\left(\sum_{j=0}^{n} \lambda_{j}\right)^{3}
$$

Now

$$
x p^{\prime}(x)=\sum_{k=0}^{n} \sqrt{\lambda_{k}}\left(\sum_{j=0}^{k} \lambda_{j}\right) x L_{k}^{* \prime}(x)=\sum_{m=0}^{n} b_{m} L_{m}^{*}(x),
$$

where by the recurrence formula $(2.12)$

$$
\begin{aligned}
b_{m} & =\lambda_{m} \sqrt{\lambda_{m}} \sum_{j=0}^{m} \lambda_{j}+\sqrt{1+2 \lambda_{m}} \sum_{k=m+1}^{n} \sqrt{\lambda_{k}\left(1+2 \lambda_{k}\right)} \sum_{j=0}^{k} \lambda_{j} \\
& \geq \sqrt{\lambda_{m}} \sum_{k=m}^{n} \lambda_{k} \sum_{j=0}^{k} \lambda_{j} \geq 0 .
\end{aligned}
$$


Hence

$$
\begin{aligned}
\int_{0}^{1}\left|x p^{\prime}(x)\right|^{2} d x & =\sum_{m=0}^{n}\left|b_{m}\right|^{2} \geq \sum_{m=0}^{n} \lambda_{m}\left(\sum_{k=m}^{n} \lambda_{k} \sum_{j=0}^{k} \lambda_{j}\right)^{2} \\
& =\sum_{\substack{0 \leq m \leq n \\
m \leq k, k^{\prime} \leq n \\
0 \leq j \leq j^{\prime} \leq k^{\prime}}} \sum_{\substack{0 \leq j \\
0, \lambda_{k} \lambda_{j} \lambda_{k^{\prime}} \lambda_{j^{\prime}}}} \sum \sum_{0 \leq m \leq j \leq j^{\prime} \leq k \leq k^{\prime} \leq n} \lambda_{m} \lambda_{k} \lambda_{j} \lambda_{k^{\prime}} \lambda_{j^{\prime}} \geq \frac{1}{5 !}\left(\sum_{k=0}^{n} \lambda_{k}\right)^{5} .
\end{aligned}
$$

This, together with (3.10), proves (3.9), and hence the left-hand side of the inequality (3.8).

We believe that the general $L^{p}$ analogue of Theorem 3.4 is true. When the sequence $\left\{\lambda_{0}, \lambda_{1}, \lambda_{2}, \ldots\right\}$ is lacunary, the proof can be obtained from (5.10) in Lemma 5.6.

\section{ON ZEROES OF MÜNTZ-LEGENDRE POLYNOMIALS}

In this section we always assume that $\lambda_{0}, \lambda_{1}, \ldots, \lambda_{n}$ are real numbers (not necessarily distinct) greater than $-1 / 2$. We make several observations on the zeros of Müntz-Legendre polynomials, some of them are interesting for their own right. The main result is a lexicographic property of the zeros given by Theorem 4.9 .

Proposition 4.1. For a function $f \in C(0,1)$, let $S^{-}(f)$ and $Z(f)$ denote the number of sign changes and the number of zeros, respectively, of $f$ in $(0,1)$ (in the count we count the zeros where there is no sign change, twice). Let $\Phi$ and $\Psi$ be in $C(0,1)$. If there is an $n$, such that

$$
n \leq S^{-}(\alpha \Phi+\beta \Psi) \leq Z(\alpha \Phi+\beta \Psi) \leq n+1
$$

holds for every real $\alpha$ and $\beta$ with $\alpha^{2}+\beta^{2}>0$, then the zeros of $\Phi$ and $\Psi$ strictly interlace.

A proof may be found in [19, Theorem 1.1 and Corollary 2].

Proposition 4.2. Assume that

$$
\left\{\lambda_{0}, \lambda_{1}, \ldots, \lambda_{n}\right\}=\left\{\tilde{\lambda}_{0}, \tilde{\lambda}_{1}, \ldots, \tilde{\lambda}_{m}\right\}
$$

where the numbers

$$
\tilde{\lambda}_{0}, \tilde{\lambda}_{1}, \ldots, \tilde{\lambda}_{m}
$$

are distinct, and let $m_{j}, j=0,1, \ldots, m$, be the number of indices $i=$ $0,1, \ldots, n$ for which $\lambda_{i}=\tilde{\lambda}_{j}$. Then $L_{n}\left(\lambda_{0}, \ldots, \lambda_{n}\right)$ is in the Chebyshev space

$$
H_{n}:=\operatorname{span}\left\{x^{\lambda_{j}}(\log x)^{i}: j=0,1, \ldots, m, i=0,1, \ldots, m_{j}-1\right\} \text {. }
$$

This follows from the definition (cf. (2.5)) and the residue theorem.

Proposition 4.3. $\left\{L_{k}\left(\lambda_{0}, \ldots, \lambda_{k}\right)\right\}_{k=0}^{n}$ is a basis of the Chebyshev space $H_{n}$ defined in Proposition 4.2.

This follows from orthogonality (cf. Theorem 2.4). 
Proposition 4.4. $L_{n}=L_{n}\left(\lambda_{0}, \ldots, \lambda_{n}\right)$ has exactly $n$ distinct zeros in $(0,1)$, and $L_{n}$ changes sign at each of these zeros.

Proof. Assume to the contrary that the number of sign changes of $L_{n}$ in $(0,1)$ is less than $n$. By Proposition 4.3, there is a function $p \in \operatorname{span}\left\{L_{k}\right\}_{k=0}^{n-1}$, which changes sign exactly at those points in $(0,1)$ where $L_{n}$ changes sign. Then $\int_{0}^{1} L_{n} p \neq 0$ which contradicts Theorem 2.4.

Proposition 4.5. Let $\lambda_{n}<\lambda_{n}^{*}$. Then the zeros of

$$
\Phi=L_{n}\left(\lambda_{0}, \ldots, \lambda_{n-1}, \lambda_{n}\right)
$$

and

$$
\Psi=L_{n}\left(\lambda_{0}, \ldots, \lambda_{n-1}, \lambda_{n}^{*}\right)
$$

in $(0,1)$ strictly interlace.

Proof. Note that Theorem 2.4 implies

$$
\int_{0}^{1}(\alpha \Phi+\beta \Psi) p=0
$$

for every $p \in H_{n-1}$, where $H_{n-1}$ is defined in Proposition 4.2. As in the proof of Proposition 4.4, $\alpha \Phi+\beta \Psi$ has at least $n$ sign changes in $(0,1)$, whenever $\alpha$ and $\beta$ are real with $\alpha^{2}+\beta^{2}>0$. Proposition 4.2 implies that $\alpha \Phi+\beta \Psi$ cannot have more than $n+1$ zeros in $(0,1)$ whenever $\alpha$ and $\beta$ are real with $\alpha^{2}+\beta^{2}>0$. Now the proof can be finished by Proposition 4.1.

Proposition 4.6. Let $\lambda_{0}, \ldots, \lambda_{k-1}, \lambda_{k+1}, \ldots, \lambda_{n}$ be fixed distinct numbers. Suppose $\left\{\lambda_{k, i}\right\}_{i=1}^{\infty} \subset(-1 / 2, \infty)$ is a sequence with $\lim _{i \rightarrow \infty} \lambda_{k, i}=\infty$. Then the largest zero of

$$
L_{n, k, i}=L_{n}\left(\lambda_{0}, \ldots, \lambda_{k-1}, \lambda_{k, i}, \lambda_{k+1}, \ldots, \lambda_{n}\right)
$$

in $(0,1)$ tends to 1 .

In particular, for the functions $\Phi$ and $\Psi$ of Proposition 4.5, the jth largest zero of $\Phi$ in $(0,1)$ is less than the jth largest zero of $\Psi$ in $(0,1)$ for every $j=1,2, \ldots, n$.

Proof. Assume, without loss of generality, that $\lambda_{k, i}$ is greater than each of the numbers $\lambda_{j}, j=0,1, \ldots, n, j \neq k$. We distinguish two cases.

Case 1. $k=n$. Let

$$
g_{i}(x)=\lambda_{n, i}\left(L_{n, n, i}(x)-c_{n, n}^{(i)} x^{\lambda_{n, i}}\right),
$$

where

$$
c_{n, n}^{(i)}=\frac{\prod_{j=0}^{n-1}\left(\lambda_{n, i}+\lambda_{j}+1\right)}{\prod_{j=0}^{n-1}\left(\lambda_{n, i}-\lambda_{j}\right)}
$$

is the coefficient of $x^{\lambda_{n, i}}$ in $L_{n, n, i}$. Now Corollary 2.2 implies that the functions $g_{i}$ converge uniformly on $[\delta, 1], \delta \in(0,1)$, to a function

$$
0 \not \equiv g \in H_{n-1}=\operatorname{span}\left\{x^{\lambda_{0}}, x^{\lambda_{1}}, \ldots, x^{\lambda_{n-1}}\right\} .
$$

By using $L_{n, n, i}(1)=1$ (cf. Corollary 2.7), and the explicit formula for $c_{n, n}^{(i)}$, it follows that $g(1) \leq 0$ and

$$
\lambda_{n, i} L_{n, n, i}(x)=\lambda_{n, i}\left(L_{n, n, i}(x)-c_{n, n}^{(i)} x^{\lambda_{n, i}}\right)+\lambda_{n, i} c_{n, n}^{(i)} x^{\lambda_{n, i}}
$$


converge to $g(x)$, as $i \rightarrow \infty$, for every $x \in(0,1)$.

Now assume that the statement of the proposition is false. Then there is an $\varepsilon \in(0,1)$ and a subsequence $\left\{\lambda_{n, i_{j}}\right\}_{j=1}^{\infty}$ of $\left\{\lambda_{n, i}\right\}_{i=1}^{\infty}$ so that the MüntzLegendre polynomials $L_{n, n, i_{j}}$ have no zeros in [1- $\left.\varepsilon, 1\right]$. It now follows that $L_{n, n, i}$ is nondecreasing on $[1-\varepsilon, 1]$, otherwise $L_{n, n, i}^{\prime}(1)>0$ (cf. Corollary 2.7) would imply that $L_{n, n, i}^{\prime}$ has at least $n+1$ zeros in $(0,1)$, which is impossible. This yields that $g$ is nondecreasing in $[1-\varepsilon, 1]$. Since $0 \not \equiv g \in$ $H_{n-1}$ and $g(1) \leq 0, g(1-\varepsilon)<0$. Therefore, $L_{n, n, i}(1-\varepsilon)<0$ if $i$ is large enough. Since $L_{n, n, i}(1)=1$ (cf. Corollary 2.7), each $L_{n, n, i}$ has a zero in $(1-\varepsilon, 1)$ if $i \geq i_{0}$, which contradicts our assumption.

Case 2. $0 \leq k \leq n-1$. Let

$$
g_{i}(x)=\lambda_{k, i}\left(L_{n, k, i}(x)-c_{k, n}^{(i)} x^{\lambda_{k, i}}\right)
$$

where

$$
c_{k, n}^{(i)}=\frac{\prod_{j=0}^{n-1}\left(\lambda_{k, i}+\lambda_{j}+1\right)}{\prod_{j=0, j \neq k}^{n}\left(\lambda_{k, i}-\lambda_{j}\right)}
$$

is the coefficient of $x^{\lambda_{k, i}}$ in $L_{n, k, i}$. From Corollary 2.2, we can deduce that the functions $g_{i}$ converge uniformly on $[\delta, 1], \delta \in(0,1)$, to a function

$$
0 \not \equiv g \in H_{n-1}=\operatorname{span}\left\{x^{\lambda_{0}}, \ldots, x^{\lambda_{k-1}}, x^{\lambda_{k+1}}, \ldots, x^{\lambda_{n}}\right\} \text {. }
$$

By using $L_{n, k, i}(1)=1$ (cf. Corollary 2.7) and the explicit formula for $c_{k, n}^{(i)}$, it follows that $g(1) \leq 0$ and

$$
L_{n, k, i}(x)=\left(L_{n, k, i}(x)-c_{k, n}^{(i)} x^{\lambda_{k, i}}\right)+c_{k, n}^{(i)} x^{\lambda_{k, i}}
$$

converge to $g(x)$, as $i \rightarrow \infty$, for every $x \in(0,1)$. Now the proof of Case 2 can be finished as in Case 1.

The second part of the proposition follows from Case 1 and the interlacing properties of zeros (cf. Proposition 4.5) by a limiting argument.

Proposition 4.7. Let $\lambda_{k} \neq \lambda_{n}$. Then the zeros of

$$
\Phi=L_{n}\left(\lambda_{0}, \ldots, \lambda_{k-1}, \lambda_{k}, \lambda_{k+1}, \ldots, \lambda_{n-1}, \lambda_{n}\right)
$$

and

$$
\Psi=L_{n}\left(\lambda_{0}, \ldots, \lambda_{k-1}, \lambda_{n}, \lambda_{k+1}, \ldots, \lambda_{n-1}, \lambda_{k}\right)
$$

in $(0,1)$ strictly interlace.

Proof. Using arguments similar to those in the proof of Proposition 4.5, it is easy to see that

$$
n-1 \leq S^{-}(\alpha \Phi+\beta \Psi) \leq Z(\alpha \Phi+\beta \Psi) \leq n,
$$

and Proposition 4.1 gives the conclusion. The observations $\Phi(1)=\Psi(1)=1$ and $\Psi^{\prime}(1)-\Phi^{\prime}(1)=\lambda_{n}-\lambda_{k} \neq 0$ (cf. Corollary 2.7) guarantee that $\alpha \Phi+\beta \Psi \not \equiv 0$ whenever $\alpha$ and $\beta$ are real with $\alpha^{2}+\beta^{2}>0$.

Proposition 4.8. Let $\Phi$ and $\Psi$ be as in Proposition 4.7. Let $x_{1}<x_{2}<\cdots<x_{n}$ and $x_{1}^{*}<x_{2}^{*}<\cdots<x_{n}^{*}$ be the zeros of $\Phi$ and $\Psi$ in $(0,1)$. Then $\lambda_{k}<\lambda_{n}$ implies that $x_{j}<x_{j}^{*}, j=1,2, \ldots, n$.

Proof. By Proposition 4.7, it is sufficient to prove that $x_{n}<x_{n}^{*}$. Let $H_{n}$ be the Chebyshev space defined in Proposition 4.2. Corollary 2.7 implies $\Phi(1)=$ 
$\Psi(1)=1$ and $\Psi^{\prime}(1)-\Phi^{\prime}(1)=\lambda_{n}-\lambda_{k}>0$. From this, and Proposition 4.7, we can deduce that $x_{n} \geq x_{n}^{*}$ would imply that $0 \not \equiv \Psi-\Phi \in H_{n}$ has at least $n+1$ distinct zeros in $(0,1]$, which is a contradiction.

Theorem 4.9. Suppose $\max \left\{\lambda_{j}: 1 \leq j \leq n\right\} \leq \min \left\{\mu_{j}: 1 \leq j \leq n\right\}$ and $\lambda_{i}<\mu_{j}$ for some $i$ and $j$. Let $x_{1}<x_{2}<\cdots<x_{n}$ and $x_{1}^{*}<x_{2}^{*}<\cdots<x_{n}^{*}$ be the zeros of $L_{n}\left(\lambda_{0}, \ldots, \lambda_{n}\right)$ and $L_{n}\left(\mu_{0}, \ldots, \mu_{n}\right)$, respectively, in $(0,1)$. Then $x_{j}<x_{j}^{*}$, $j=1,2, \ldots, n$.

Proof. Repeated applications of Propositions 4.6 and 4.8 yield the desired result.

Proposition 4.10. Let $\lambda_{0}<\lambda_{n}$. Let $x_{1}<x_{2}<\cdots<x_{n}$ and $x_{1}^{*}<x_{2}^{*}<\cdots<$ $x_{n}^{*}$ be the zeros of $L_{n}\left(\lambda_{0}, \lambda_{1}, \ldots, \lambda_{n}\right)$ and $L_{n}\left(\lambda_{n}, \lambda_{n-1}, \ldots, \lambda_{0}\right)$, respectively, in $(0,1)$. Then $\left\{x_{j}\right\}_{j=1}^{n}$ and $\left\{x_{j}^{*}\right\}_{j=1}^{n}$ strictly interlace and $x_{j}<x_{j}^{*}, j=$ $1,2, \ldots, n$.

Proof. This follows from Propositions 4.7 and 4.8 and the Remark given after Corollary 2.2.

Proposition 4.11. The zeros of

$$
\Phi=L_{n-1}\left(\lambda_{0}, \ldots, \lambda_{n-1}\right) \text { and } \Psi=L_{n}\left(\lambda_{0}, \ldots, \lambda_{n}\right)
$$

in $(0,1)$ strictly interlace.

Proof. Proposition 4.1 and arguments similar to those given in the proof of Proposition 4.5 yield the theorem.

Corollary 4.12. Assume that $x_{1}<x_{2}<\cdots<x_{n}$ are the zeros of $L_{n}\left(\lambda_{0}, \ldots, \lambda_{n}\right)$ in $(0,1)$. Then,

$$
\exp \left(-\frac{4 n+2}{1+2 \lambda_{*}}\right)<x_{1}<x_{2}<\cdots<x_{n}<\exp \left(\frac{-j_{1}^{2}}{\left(1+2 \lambda^{*}\right)(4 n+2)}\right),
$$

where $\lambda_{*}:=\min \left\{\lambda_{0}, \ldots, \lambda_{n}\right\}, \lambda^{*}:=\max \left\{\lambda_{0}, \ldots, \lambda_{n}\right\}$ and $j_{1}>3 \pi / 4$ is the smallest positive zero of the Bessel function $J_{0}(z)=\sum_{k=0}^{\infty}\left(-z^{2}\right)^{k} /\left(k ! 2^{k}\right)^{2}$.

Proof. Let $\mathscr{L}_{n}$ be the $n$th Laguerre polynomial with respect to the weight $e^{-x}$ on $[0, \infty)$, and let the zeros of $\mathscr{L}_{n}$ be $z_{1}<z_{2}<\cdots<z_{n}$. Then we have (cf. $[24$, pp. $127-131]$ )

$$
\frac{j_{1}^{2}}{4 n+2}<z_{1}<z_{2}<\cdots<z_{n}<4 n+2,
$$

where the upper estimate is asymptotically sharp, and the lower estimate is sharp up to a constant (not exceeding $4^{4} / 9 \pi^{2}$ ). Since $n$ is fixed, we let $\varepsilon>0$ be sufficiently small that $\lambda_{*}-n \varepsilon>-1 / 2$. Then all the zeros of $L_{n}\left(\lambda_{0}, \ldots, \lambda_{n}\right)$ lie to the right of those of $L_{n}\left(\lambda_{*}, \lambda_{*}-\varepsilon, \ldots, \lambda_{*}-n \varepsilon\right)$ by Theorem 4.9. From the contour integral formula $(2.5), L_{n}\left(\lambda_{*}, \lambda_{*}-\varepsilon, \ldots, \lambda_{*}-n \varepsilon\right)$ tends to $L_{n}\left(\lambda_{*}, \lambda_{*}, \ldots, \lambda_{*}\right)$ uniformly on closed subintervals of $(0,1]$ as $\varepsilon \rightarrow 0$. Recalling that (cf. Corollary 2.3 )

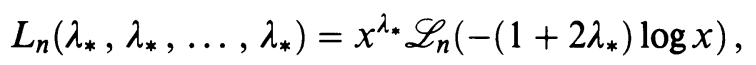

we conclude that $x_{1} \geq y_{1}$, where $y_{1}$ is the smallest zero of $L_{n}\left(\lambda_{*}, \lambda_{*}, \ldots, \lambda_{*}\right)$. Since $z_{n}=-\left(1+2 \lambda_{*}\right) \log y_{1}$, we can combine this with (4.1) to get

$$
x_{1} \geq y_{1}=\exp \left(\frac{-z_{n}}{1+2 \lambda_{*}}\right)>\exp \left(-\frac{4 n+2}{1+2 \lambda_{*}}\right) \text {, }
$$


which is the left-hand side inequality of this corollary. It can be seen similarly that all zeros of $L_{n}\left(\lambda_{0}, \ldots, \lambda_{n}\right)$ lie to the left of the zeros of

$$
L_{n}\left(\lambda^{*}, \ldots, \lambda^{*}\right)=x^{\lambda^{*}} \mathscr{L}_{n}\left(-\left(1+2 \lambda^{*}\right) \log x\right),
$$

which implies that $x_{n}<\exp \left(-j_{1}^{2} /\left(\left(1+2 \lambda^{*}\right)(4 n+2)\right)\right)$.

\section{Christoffel FUnCtions}

Christoffel functions have been intensively studied, and their utility in the theory of orthogonal polynomials and approximation theory can be illustrated by their relation with polynomial inequalities, interpolation theory, quadrature formulae, zeros of orthogonal polynomials, etc. (cf. [14]). In this section we study the Müntz-Christoffel functions and some of their applications.

We assume that $\Lambda=\left\{\lambda_{0}, \lambda_{1}, \lambda_{2}, \ldots\right\}$ satisfies

$$
0=\lambda_{0}<\lambda_{1}<\lambda_{2}<\cdots \rightarrow+\infty \text {. }
$$

The Christoffel function for the Müntz system $M(\Lambda)$ with respect to Lebesgue measure is defined by either side of the following equality

$$
\frac{1}{\sum_{k=0}^{\infty}\left|L_{k}^{*}(x)\right|^{2}}=\inf _{p \in M(\Lambda), p(x)=1} \int_{0}^{1}|p(t)|^{2} d t,
$$

which is a well-known consequence of the orthogonality (cf. [24, p. 39]). If the infimum is taken just over $M_{n}(\Lambda)$, then we have

$$
\frac{1}{\sum_{k=0}^{n}\left|L_{k}^{*}(x)\right|^{2}}=\min _{p \in M_{n}(\Lambda), p(x)=1} \int_{0}^{1}|p(t)|^{2} d t,
$$

and either side can be called the $n$th Christoffel function. Recalling the reproducing kernel (2.20), we see that $1 / K_{n}(x, x)$ and

$$
1 / K(x, x):=\lim _{n \rightarrow \infty} 1 / K_{n}(x, x)
$$

are what we have just defined (cf. (3.3) and (3.4)). For convenience, we will defy the section title a little by stating results in terms of the reciprocal of the Christoffel functions, namely, in terms of $K(x):=K(x, x)$ and $K_{n}(x):=$ $K_{n}(x, x)$.

The classical Müntz theorem characterizes the denseness of $M(\Lambda)$ by the divergence of the series $\sum_{k=1}^{\infty} \lambda_{k}^{-1}$. Now we can connect the Christoffel functions with the denseness. All results here are stated for sequences $\Lambda$ of integers, but they hold for sequences $\Lambda$ of nonnegative real numbers.

Theorem 5.1. Let $\Lambda=\left\{0=\lambda_{0}<\lambda_{1}<\cdots\right\}$ be an integer sequence. Then the following statements are equivalent:

(i) $M(\Lambda)$ is not dense in $C[0,1]$ in the uniform norm;

(ii) $\sum_{k=1}^{\infty} \lambda_{k}^{-1}<+\infty$;

(iii) There is an $x \in[0,1)$, such that $\sum_{k=0}^{\infty}\left|L_{k}^{*}(x)\right|^{2}<+\infty$;

(iv) $\sum_{k=0}^{\infty}\left|L_{k}^{*}(x)\right|^{2}$ converges uniformly on $[0,1-\varepsilon]$ for every $0<\varepsilon<1$.

The right endpoint 1 is quite different, where we always have (cf. (2.9) and (2.14)) $K(1)=\sum_{k=0}^{\infty}\left|L_{k}^{*}(1)\right|^{2}=\sum_{k=0}^{\infty}\left(1+2 \lambda_{k}\right)=+\infty$. The following lemma is extracted from the proof of [7, Theorem 3], see also [2, Lemma 2]. It estimates the function values and derivative values of $\Lambda$-polynomials on $[0,1-\varepsilon]$ by their $L^{2}[0,1]$ norms. The proof of Theorem 5.1 will follow this. 
Lemma 5.2. Let $\Lambda=\left\{0=\lambda_{0}<\lambda_{1}<\cdots\right\}$ be an integer sequence with $\sum_{k=1}^{\infty} \lambda_{k}^{-1}$ $<+\infty$. Then there is a constant $C=C(\Lambda, \varepsilon, \nu)$ depending only on $\Lambda, \varepsilon$, and $\nu$

$$
\max _{x \in[0,1-\varepsilon]}\left|p^{(\nu)}(x)\right| \leq C\left(\int_{0}^{1}|p(x)|^{2}\right)^{1 / 2},
$$

for every $p \in M(\Lambda)$, for every $\nu=0,1,2, \ldots$, and $0<\varepsilon<1$.

Proof. Since $\Lambda$ is an integer sequence, and $\sum_{k=1}^{\infty} \lambda_{k}^{-1}<+\infty$, by the proof of [7, Theorem 3], for every $\varepsilon>0$, there is constant $C_{0}=C_{0}(\Lambda, \varepsilon)>0$ depending only on $\Lambda$ and $\varepsilon$ such that

$$
\left|a_{k}\right| \leq C_{0}(1+\varepsilon)^{\lambda_{k}}\left(\int_{0}^{1}|p(x)|^{2} d x\right)^{1 / 2}, \quad k=0,1,2, \ldots,
$$

hold for every $\Lambda$-polynomial $p(x)=\sum_{k=0}^{n} a_{k} x^{\lambda_{k}}$, and for every $n=0,1,2, \ldots$. (We remark that the above also holds if $\inf _{k \in \mathbb{N}}\left\{\lambda_{k}-\lambda_{k-1}\right\}>0$.) Note in particular that $C_{0}$ is independent of $n$. Hence

$$
\left|p^{(\nu)}(x)\right| \leq \sum_{k=\nu}^{n}\left|a_{k}\right| \lambda_{k}^{\nu} x^{\lambda_{k}-\nu} \leq C_{0} \sum_{k=\nu}^{n}(1+\varepsilon)^{\lambda_{k}}\left(\int_{0}^{1}|p(x)|^{2} d x\right)^{1 / 2} \lambda_{k}^{\nu} x^{\lambda_{k}-\nu}
$$

If $x \in[0,1-\varepsilon]$, then $(1+\varepsilon) x \leq 1-\varepsilon^{2}$, and the above implies that

$$
\left|p^{(\nu)}(x)\right| \leq C_{0}(1+\varepsilon)^{\nu} \sum_{k=0}^{\infty}\left(1-\varepsilon^{2}\right)^{k} k^{\nu}\left(\int_{0}^{1}|p(t)|^{2} d t\right)^{1 / 2} .
$$

Therefore, (5.4) holds with $C(\Lambda, \varepsilon, \nu)=C_{0}(1+\varepsilon)^{\nu} \sum_{k=0}^{\infty}\left(1-\varepsilon^{2}\right)^{k} k^{\nu}$.

A simple consequence of the above is a bounded Nikolskii-type inequality.

Corollary 5.3. Under the condition of Lemma 5.2,

$$
\max _{x \in[0,1-\varepsilon]}|p(x)| \leq C \int_{0}^{1}|p(x)| d x, \quad p \in M(\Lambda),
$$

where $C=C(\Lambda, \varepsilon)$ depends only on $\Lambda$ and $\varepsilon$.

Proof. Consider the new sequence $\Lambda^{*}=\left\{1, \lambda_{0}+1, \lambda_{1}+1, \ldots\right\}$ and the Müntz space $M\left(\Lambda^{*}\right)=\operatorname{span}\left\{1, x^{\lambda_{0}+1}, x^{\lambda_{1}+1}, \ldots\right\}$. Apply Lemma 5.2 with $\nu=1$ for the $\Lambda^{*}$-polynomials $\int_{0}^{x} p(t) d t$ with $p \in M(\Lambda)$, and use the simple fact that $\left|\int_{0}^{x} p(t) d t\right| \leq \int_{0}^{1}|p(t)| d t$.

Proof of Theorem 5.1. The equivalence of (i) and (ii) is the classical MüntzSzász Theorem. We show that (ii) $\Rightarrow$ (iv) $\Rightarrow$ (iii) $\Rightarrow$ (i).

(ii) $\Rightarrow$ (iv). Since $\sum_{k=0}^{\infty} \lambda_{k}^{-1}<+\infty$, we have by Corollary 3.2 that

$$
\sum_{k=0}^{\infty}\left|L_{k}^{*(\nu)}(x)\right|^{2}=\sup \left\{\left|p^{(\nu)}(x)\right|^{2}: p \in M(\Lambda), \int_{0}^{1}|p(x)|^{2} d x=1\right\}
$$

for every $x \in[0,1]$. Hence by Lemma 5.2 , for every $\varepsilon>0$ there is a constant $C=C(\Lambda, \varepsilon)$ such that

$$
\sum_{k=0}^{\infty}\left|L^{*}(x)\right|^{2} \leq C, \quad \sum_{k=0}^{\infty}\left|L^{*} \prime(x)\right|^{2} \leq C, \quad x \in[0,1-\varepsilon] .
$$


Since

$$
\frac{d}{d x} \sum_{k=0}^{n}\left(L^{*}(x)\right)^{2}=\sum_{k=0}^{n} L_{k}^{*}(x) L_{k}^{*} \prime(x)
$$

on applying the Cauchy-Schwarz inequality and (5.6), we see that (5.7) is uniformly bounded by $C$ for $x \in[0,1-\varepsilon]$, and $n \geq 0$. Therefore the functions $\sum_{k=0}^{n}\left|L^{*}(x)\right|^{2}$ are equicontinuous on $[0,1-\varepsilon]$ for $n=0,1,2, \ldots$, which implies the uniform convergence of $K_{n}$ to $K$ on $[0,1-\varepsilon]$ by the Arzela-Ascoli Theorem.

The implication (iv) $\Rightarrow$ (iii) is trivial.

We now finish the proof by showing (iii) $\Rightarrow$ (i). Assume that $K\left(x_{0}\right)<+\infty$ for some $x_{0} \in[0,1]$. Then $M(\Lambda)$ fails to be dense in $C[0,1]$. Otherwise, let $f \in C[0,1]$ be such that $\left|f\left(x_{0}\right)\right|^{2} \geq K\left(x_{0}\right)+2$ and $\int_{0}^{1}|f(x)|^{2}=1$. Then by the density assumption, there is a $p \in M(\Lambda)$, such that $\left|p\left(x_{0}\right)\right|^{2} \geq K\left(x_{0}\right)+1$ and $\int_{0}^{1}|p(x)|^{2} d x=1$, which means that

$$
\sup \left\{\left|p\left(x_{0}\right)\right|: p \in M(\Lambda), \int_{0}^{1}|p(x)|^{2} d x=1\right\} \geq K\left(x_{0}\right)+1
$$

which contradicts (5.3).

Actually when $M(\Lambda)$ is not dense, the uniform convergence also holds for higher derivatives, and in this case, we do not assume $\lambda_{0}=0$.

Theorem 5.4. Let $\Lambda=\left\{0 \leq \lambda_{0}<\lambda_{1}<\cdots\right\}$ be a sequence of integers with $\sum_{k=1}^{\infty} \lambda_{k}^{-1}<+\infty$. Then

$$
\sum_{k=0}^{\infty}\left|L_{k}^{*(\nu)}(x)\right|^{2} \text { converges uniformly on }[0,1-\varepsilon]
$$

for every $\nu=0,1,2, \ldots$ and every $0<\varepsilon<1$.

Proof. The method is exactly the same as in the proof of (ii) $\Rightarrow$ (iv) of Theorem 5.1. Lemma 5.2 implies the uniform boundedness of the series in (5.8) and that of $\sum_{k=0}^{\infty}\left|L_{k}^{*(\nu+1)}\right|^{2}$ on $[0,1-\varepsilon]$, and the uniform boundedness of $\frac{d}{d x} \sum_{k=0}^{n}\left|L_{k}^{*(\nu)}(x)\right|^{2}$ on $[0,1-\varepsilon]$ follows by Cauchy-Schwarz inequality. Now the Arzela-Ascoli Theorem completes the proof.

We obtain immediately from Theorem 5.4 that under the conditions of Theorem 5.4, the orthonormal Müntz-Legendre polynomials tend to 0 uniformly on closed subintervals of $[0,1)$. Whereas for orthogonal polynomials $p_{n}, n=0,1,2, \ldots$, orthonormal with respect to a measure supported on $[0,1]$, only the relative growth $\left|p_{n}\right|^{2} / \sum_{k=0}^{n}\left|p_{k}\right|^{2}$ tends to 0 uniformly on $[0,1]$ (cf. $[15,16,27])$.

Corollary 5.5. Suppose $0=\lambda_{0}<\lambda_{1}<\cdots \rightarrow \infty$ is a sequence of integers, and the associated Müntz system is not dense in $C[0,1]$. Then

$$
\lim _{k \rightarrow \infty} \max _{x \in[0,1-\varepsilon]}\left|L_{k}^{*(\nu)}(x)\right|=0
$$

holds for every $0<\varepsilon<1$ and every $\nu=0,1,2, \ldots$.

When the sequence $\Lambda$ is lacunary, that is,

$$
\inf \left\{\lambda_{k+1} / \lambda_{k}: k=0,1,2, \ldots\right\}>1,
$$


we can say more about the boundedness of the function $K$. To do this, we first give a bounded Bernstein-type and a bounded Nikolskii-type inequality for a lacunary system (cf. [4, Theorem 3.1]).

Lemma 5.6. Let $\Lambda=\left\{0=\lambda_{0}<\lambda_{1}<\lambda_{2}<\cdots\right\}$ be lacunary as in (5.9). Then

$$
\left|p^{\prime}(x)\right| \leq \frac{C}{1-x} \max _{t \in[0,1]}|p(t)|, \quad x \in[0,1), p \in M(\Lambda),
$$

and

$$
|p(x)| \leq \frac{C}{1-x} \int_{0}^{1}|p(t)| d t, \quad x \in[0,1), p \in M(\Lambda),
$$

hold with a constant $C=C(\Lambda)$ depending only on $\Lambda$.

Proof. The inequality (5.10) comes from [4, Theorem 3.1]. For (5.11), consider the new lacunary sequence $\Lambda^{*}=\left\{0,1+\lambda_{0}, 1+\lambda_{1}, \ldots\right\}$, and apply (5.10) for $\Lambda^{*}$-polynomials which are indefinite integrals of $p \in M(\Lambda)$.

Theorem 5.7. Let $\Lambda=\left\{\lambda_{0}, \lambda_{1}, \lambda_{2}, \ldots\right\}$ be lacunary. Then there is a constant $C=C(\Lambda)$ depending only on $\Lambda$, such that

$$
K(x)=\sum_{k=0}^{\infty}\left|L_{k}^{*}(x)\right|^{2} \leq \frac{C}{(1-x)^{2}}, \quad x \in[0,1) .
$$

Proof. Since $\Lambda$ is lacunary, applying Lemma 5.6, we get

$$
|p(x)|^{2} \leq \frac{C}{(1-x)^{2}}\left(\int_{0}^{1}|p(t)| d t\right)^{2} \leq \frac{C}{(1-x)^{2}} \int_{0}^{1}|p(t)|^{2} d t .
$$

By (5.2)-(5.3) or (3.2)-(3.4), we have $K(x) \leq C /(1-x)^{2}$.

As a last observation in this paper, we point out that if $\lambda_{n} \rightarrow \infty$, then there are numbers $x_{n} \rightarrow 1^{-}$, such that $K\left(x_{n}\right) \geq C_{1} /\left(1-x_{n}\right)$. Indeed, let $x_{n}=1-1 / \lambda_{n}$, and consider $p(x)=x^{\lambda_{n}}$. Then by (5.2)-(5.3) or Corollary 3.2,

$$
\begin{aligned}
K\left(x_{n}\right) & \geq p\left(x_{n}\right)^{2} /\|p\|_{2}^{2}=x_{n}^{2 \lambda_{n}}\left(2 \lambda_{n}+1\right) \\
& =\left(1-1 / \lambda_{n}\right)^{2 \lambda_{n}}\left(2 \lambda_{n}+1\right) \geq C_{1} \lambda_{n} \geq C_{1} /\left(1-x_{n}\right),
\end{aligned}
$$

where $C_{1}=\inf \left\{\left(1-1 / \lambda_{n}\right)^{2 \lambda_{n}}: n=1,2,3, \ldots\right\}>0$.

\section{REFERENCES}

1. J. M. Anderson, Müntz-Szász type approximation and the angular growth of $L$ integral functions, Trans. Amer. Math. Soc. 169 (1972), 237-248.

2. P. B. Borwein, Zeros of Chebyshev polynomials in Markov systems, J. Approx. Theory 63 (1990), 56-64.

3. P. B. Borwein and T. Erdélyi, Lacunary Müntz systems, J. Edinburgh Math. Soc. (to appear).

4. , Notes on lacunary Müntz polynomials, Israel J. Math. 76 (1991), 183-192.

5. P. B. Borwein and E. B. Saff, On the denseness of weighted incomplete approximations, Progress in Approximation Theory (A. Gonchar and E. B. Saff, eds.), Springer-Verlag, 1992, pp. 419-429.

6. E. W. Cheney, Introduction to approximation theory, McGraw-Hill, New York, 1966.

7. J. A. Clarkson and P. Erdős, Approximation by polynomials, Duke Math. J. 10 (1943), 5-11. 
8. R. P. Feinerman and D. J. Newman, Polynomial approximation, Williams and Wilkins, Baltimore, Md., 1976.

9. G. Freud, Orthogonal polynomials, Pergamon Press, Oxford, 1971.

10. M. von Golitschek, A short proof of Müntz theorem, J. Approx. Theory 39 (1983), 394-395.

11. D. Leviatan, Improved estimates in Müntz-Jackson theorems, Progress in Approximation Theory (P. Nevai and A. Pinkus, eds.), Academic Press, 1991, pp. 567-573.

12. G. G. Lorentz, Approximation by incomplete polynomials (problems and results), Padé and Rational Approximation, Theory and Applications (E. B. Saff and R. S. Varga, eds.), Academic Press, New York, 1977.

13. P. C. McCarthy, J. E. Sayre, and B. L. R. Shawyer, Generalized Legendre polynomials, manuscript, 1989.

14. P. Nevai, Géza Freud, orthogonal polynomials and Christoffel functions. A case study, J. Approx. Theory 48 (1986), 3-167.

15. P. Nevai, V. Totik, and J. Zhang, Orthogonal polynomials: their growth relative to their sums, J. Approx. Theory 67 (1991), 215-234.

16. P. Nevai and J. Zhang, Rate of relative growth of orthogonal polynomials, J. Math. Anal. Appl. 175 (1993), 10-24.

17. D. J. Newman, Derivative bounds for Müntz polynomials, J. Approx. Theory 18 (1976), 360-362.

18. __ Approximation with rational functions, CBMS Regional Conf. Ser. in Math., vol. 41, Amer. Math. Soc., Providence, R.I., 1978.

19. A. Pinkus and Z. Ziegler, Interlacing properties of zeros of the error functions in best $L^{p}$ approximations, J. Approx. Theory 27 (1979), 1-18.

20. E. B. Saff and R. S. Varga, On incomplete polynomials, Numerische Methoden der Approximationstheorie (L. Collatz, G. Meinardus, and H. Weiner, eds.), Birkhäuser-Verlag, Basel, 1978.

21. L. Schwartz, Etude des sommes d'exponentielles, Hermann, Paris, 1959.

22. P. W. Smith, An improvement theorem for Descartes systems, Proc. Amer. Math. Soc. 70 (1978), 26-30.

23. G. Somorjai, A Müntz-type problem for rational approximation, Acta Math. Hungar. 27 (1976), 197-199.

24. G. Szegö, Orthogonal polynomials, Amer. Math. Soc. Colloq. Publ., vol. 23, 4th ed., Amer. Math. Soc., Providence, R.I., 1975.

25. A. K. Taslakyan, Some properties of Legendre quasi-polynomials with respect to a Müntz system, Mathematics 2, Erevan University, Erevan, 1984, pp. 179-189. (Russian, Armenian Summary)

26. T. T. Trent, $A$ Müntz-Szász theorem for $C(D)$, Proc. Amer. Math. Soc. 83 (1981), 296-298.

27. J. Zhang, Relative growth of linear iterations and orthogonal polynomials on several intervals, Linear Algebra Appl. 186 (1993), 97-115.

(Peter Borwein and Tamás Erdélyi) Department of Mathematics, Simon Fraser UniverSITY, BuRnaBY, B.C., CANADA V5A 1S6

E-mail address: pborwein@cs.sfu.ca

E-mail address: erdelyi@cs.sfu.ca

(John Zhang) SCCM, Department of Computer Science, Stanford University, Stanford, CALIFORNIA 94305

E-mail address: zhang@sccm.stanford.edu 